\title{
Laboratory techniques to obtain different forms of Trypanosoma cruzi: applications to wild-type and genetically modified parasites
}

\author{
María de los Milagros Cámara, León A. Bouvier, Mariana R. Miranda, Chantal Reigada and Claudio A. Pereira
}

Laboratorio de Biología Molecular de Trypanosoma cruzi, Instituto de Investigaciones Médicas 'Alfredo Lanari', Universidad de Buenos Aires and CONICET, Buenos Aires, Argentina

\begin{abstract}
Nowadays, there are no simple techniques for mimicking in vitro the life cycle of the kinetoplasmtid Trypanosoma cruzi Chagas, 1909, causative agent of Chagas disease, especially for parasite strains maintained as epimastigotes for many years. In the present study, we propose a method for obtaining metacyclic trypomastigotes, which were capable of infecting mammalian cells by simply lowering $\mathrm{pH}$ media. The collected amastigotes and trypomastigotes were differentiated into epimastigotes closing $T$. cruzi life cycle in vitro. Metacyclogenesis rates and infectivity were enhanced in cycled parasites. Finally, using this method, we were able to infect cells with transgenic parasites obtaining trypomastigotes and amastigotes using a neomycin-resistant cell line.
\end{abstract}

Keywords: Chagas disease, metacyclogenesis, parasite differentiation, intracellular epimastigotes

During its life cycle, the kinetoplastid Trypanosoma cruzi Chagas, 1909 suffers a wide variety of morphological and gene expression changes (Goldenberg and Avila 2011). The factors that trigger the differentiation process in T. cruzi are still unknown (Kolev et al. 2012). In nature, they occur spontaneously and can be mimicked in vitro by different techniques. However, very few of them keep the whole life cycle, returning to the epimastigote stage in each cycle.

One of the first method to differentiate epimastigotes to metacyclic trypomastigotes derives from the observation of Camargo (1964), in which about $10-15 \%$ of metacyclic forms appeared in epimastigote cultures only at the end of the exponential growth phase. The differentiation process did not occur if parasites were maintained in permanent exponential growth (Camargo 1964). A similar approach was also described by Dusanic 1980 .

A few years later, Castellani et al. (1967) proposed a method which relied on lowering $\mathrm{pH}$ medium. A reduction from 7.2 to 6.7 produced about $40 \%$ of metacyclic forms, but this process was completely abolished if $\mathrm{pH}$ was reduced to 6.3 (Castellani et al. 1967). In contrast, Ucros et al. (1983) used a defined culture medium at pH 9 to produce metacyclic forms. The most widely used method was originally published by Contreras et al. (1985); this method consists of the incubation of culture - derived epimastigotes in triatomine artificial urine (TAU), which could be supplemented with proline (TAU-P). Later, more variants were developed, such as TAU supplement- ed with proline, glutamate, aspartate and glucose (TAU$3 \mathrm{AG}$ ), which are effective for the differentiation of highly infective strains, but are not useful for laboratory cultures maintained for a long time as epimastigotes. Finally, other methods based on nutritional stress were also described (De Lima et al. 2008).

Several techniques have been developed for obtaining amastigotes in vitro. The process of amastigogenesis occurs when metacyclic trypomastigotes from triatomine urine differentiate into amastigotes inside mammalian host cells and when tissue-derived trypomastigotes invade new cells and differentiate newly to amastigotes. In vitro protocols for amastigote formation consist basically of the purification of tissue-culture trypomastigotes and amastigotes after disruption of infected Vero cells. In addition, amastigotes can be generated in vitro from tissueculture-derived trypomastigotes (Tomlinson et al. 1995, Contreras et al. 2002, Navarro et al. 2003) and also spheromastigotes can be obtained from metacyclic trypomastigotes (Rondinelli et al. 1988).

The techniques mentioned above, which are vastly used nowadays, have two main limitations: (1) there is no reliable way to complete the life cycle in vitro (Kimura et al. 1978), which implies the transformation of amastigotes in epimastigotes (De Lima et al. 2008), and (2) the majority of methods of metacyclogenesis do not work with some strains maintained for a long time in culture. Here, we present a simple and flexible protocol for obtaining all the $T$. cruzi stages emulating the natural life cycle in vitro. 


\section{MATERIALS AND METHODS}

\section{Parasites}

Stock cultures of Trypanosoma cruzi epimastigotes of the $\mathrm{CL}$ Brener and Y strains were maintained axenically at $28^{\circ} \mathrm{C}$ in Liver Infusion Triptose (LIT) or Brain Heart Triptose (BHT) media ( $\mathrm{pH} 7$ ), respectively, supplemented with $10 \%$ fetal calf serum, $100 \mathrm{U} / \mathrm{ml}$ penicillin, and $\mu \mathrm{g} / \mathrm{ml}$ streptomycin (Camargo 1964). Transfected parasites were maintained in the same media containing $100 \mu \mathrm{g} / \mathrm{ml} \mathrm{G} 418$. Parasites were counted in a Neubauer hemocytometer chamber.

\section{In vitro differentiation and infection}

Trypanosoma cruzi epimastigotes of the CL Brener and $\mathrm{Y}$ strains at stationary growing phase (15 days) were collected and grown in LIT and BHT medium at $\mathrm{pH} 4, \mathrm{pH} 5.8$ and TAU-P ( $190 \mathrm{mM} \mathrm{NaCl}, 17 \mathrm{mM} \mathrm{KCl}, 2 \mathrm{mM} \mathrm{MgCl}, 2 \mathrm{mM} \mathrm{CaCl}_{2}, 50 \mathrm{mM}$ L-proline in $8 \mathrm{mM}$ phosphate buffered saline (PBS) $\mathrm{pH}$ 6.0) for 6-8 days at $28^{\circ} \mathrm{C}$. Then, $2 \mathrm{ml}$ of culture, containing $10^{8}$ parasites (epimastigotes and trypomastigotes), were tested for cell infection using a monolayer of Vero cells $(60 \%$ confluent), grown in MEM supplemented with $10 \%$ fetal calf serum. Twenty-four hours later parasites were removed and cells were maintained at $37^{\circ} \mathrm{C}$ in MEM medium supplemented with 3\% fetal calf serum, subsequently cells' medium was replaced every day. Infections were monitored periodically under the light microscope and the number of infected cells, amastigotes/cell and released trypomastigotes/cell were recorded. Infected cells were considered those containing intracellular amastigotes. Results are presented as the average of 200 cells analysed.

Trypomastigote forms were collected after cells disruption and used for reinfection and for recycling to epimastigote stage. For epimastigotes production, trypomastigotes and amastigotes were collected and cultured in LIT or BHT medium at $\mathrm{pH} 7$. When the number of epimastigotes increased, they were subjected to differentiation at low $\mathrm{pH}$ conditions for reinfection or transfection assays. This cycle was repeated three times for the CL Brener strain and two times for the Y strain. The same procedure was followed for cycled transfected parasites. In that case, infection procedures were done in a G418 resistant Vero cells strain and selection was maintained during infection conditions.

\section{Statistics}

Data were analysed as follows: first normal distribution was tested using a Kolmogorov-Smirnov test and all groups presented a normal distribution. Afterwards, groups were analysed using a one-way ANOVA followed by a post-hoc Dunnet's multiple comparison test using a significance cut-off value of $\mathrm{P}<0.05$.

\section{Fluorescence microscopy}

Freshly grown trypanosome samples were washed twice in PBS. After letting the cells settle for $30 \mathrm{~min}$ at room temperature onto poly-L-lysine coated coverslips, parasites were fixed at room temperature for 20 min with $4 \%$ formaldehyde in PBS, followed by a cold methanol treatment for $5 \mathrm{~min}$. Afterwards, all the samples were treated with anti-GFP (green fluorescent protein) antibody (Invitrogen, Carlsbad, California, USA) for $1 \mathrm{~h}$, followed by secondary antibody incubation anti-rabbit (Vector Laboratories, Burlingame, California, USA) for $1 \mathrm{~h}$. Slides were mounted using Vectashield with DAPI (Vector Laboratories, Burlingame, California, USA).
Infected cells with transfected parasites were grown in 24-well plates. Seven day post-infection, they were washed three-times with PBS, followed by fixation at room temperature 20 min with $4 \%$ formaldehyde in PBS. Cells permeabilization was achieved by 15 min incubation with $0.01 \%(\mathrm{v} / \mathrm{v})$ Triton X-100 after fixation. Afterwards, samples were treated as explained above. Cells were observed in an Olympus BX51 fluorescence microscope. Images were recorded with an Olympus XM10 camera and analysed with MBF ImageJ for microscopy bundle (National Institutes of Health, Bethesda, Maryland, USA).

\section{Plasmid constructions and parasites transfection}

The sequence coding for the full-length T. cruzi histone H2B (TcH2B) was cloned from genomic DNA using the following primers H2BF: 5'-CCATGGCCACCCCCAAAAGCTC-3'; H2BR: 5'-GGATCCACTAGAGGCGCTGCGACACCG-3' and fused to the 3 ' end of the GFP gene present in the pTEX-eGFP expression vector by digesting EcoRI/BamHI. The pTEX-eGFP plasmid was constructed by cloning the eGFP into the pTEXTAP vector, kindly provided by Dr. Esteban Serra (Instituto de Biología Molecular y Celular de Rosario, Argentina). A total of $10^{8}$ parasites of the CL Brener strain cycled once were grown in LIT medium at $28^{\circ} \mathrm{C}$, harvested by centrifugation, washed with PBS and resuspended in $0.35 \mathrm{ml}$ of electroporation buffer (PBS containing $0.5 \mathrm{mM} \mathrm{MgCl}_{2}, 0.1 \mathrm{mM} \mathrm{CaCl}_{2}$ ). The cell suspension was mixed with $50 \mu \mathrm{g}$ of plasmid DNA in $0.2 \mathrm{~cm}$ gap cuvettes (Bio-Rad Laboratories, Hercules, California, USA). The parasites were electroporated with a single pulse of $400 \mathrm{~V}$, $500 \mu \mathrm{F}$ with a time constant of about $5 \mathrm{~ms}$. Stable cell lines were achieved after 90 days of treatment with $500 \mu \mathrm{g} / \mathrm{ml} \mathrm{G418}$ (Calbiochem, Darmstadt, Germany) and supplementation of LIT medium with $30 \%$ SFB (Pereira et al. 2003).

\section{G418 resistant Vero cells}

Stable Vero lines resistant to G418 were obtained using a pcDNA3 plasmid (Invitrogen, Carlsbad, California, USA), lacking the CMV promoter by digesting BgIII/BamHI followed by re-ligation. For cell transfection Lipofectamine (Invitrogen, Carlsbad, California, USA) was used. For each transfection assay $0.6 \mu \mathrm{g}$ of DNA were mixed with $25 \mu \mathrm{l}$ of MEM without serum or antibiotics and $25 \mu \mathrm{l}$ of Lipofectamine was added. The mixture was incubated at room temperature for $20 \mathrm{~min}$ and then placed over a Vero cell monolayer ( $70 \%$ confluent) in MEM without serum for 6 hours at cells at $37^{\circ} \mathrm{C}$ in a $\mathrm{CO}_{2}$ incubator. Cells were washed and media were replaced by MEM $10 \%$ fetal calf serum. Twenty four hours later, G418 $150 \mu \mathrm{g} / \mathrm{ml}$ was added. Cells were monitored under the light inverted microscope. Stable lines were obtained and maintained with $500 \mu \mathrm{g} / \mathrm{ml}$ of G418.

\section{RESULTS AND DISCUSSION}

Trypanosoma cruzi epimastigotes ( $10^{7}$ cells) of the CL Brener and $\mathrm{Y}$ strains, maintained by subculturing as epimastigotes for years, were placed in $5 \mathrm{ml}$ of LIT or BHT medium, respectively, at $\mathrm{pH} 4$. Four days after inoculation, intermediate forms (epimastigote/trypamastigote) with high mobility compared to trypomastigotes, and metacyclic trypomastigotes were observed. Proportion of these parasite forms increased up to $30 \%$ and $65 \%$ on day 8 , for CL Brener and Y strains, respectively. Similar treatments were also performed increasing media $\mathrm{pH}$ to 5.8 and also 

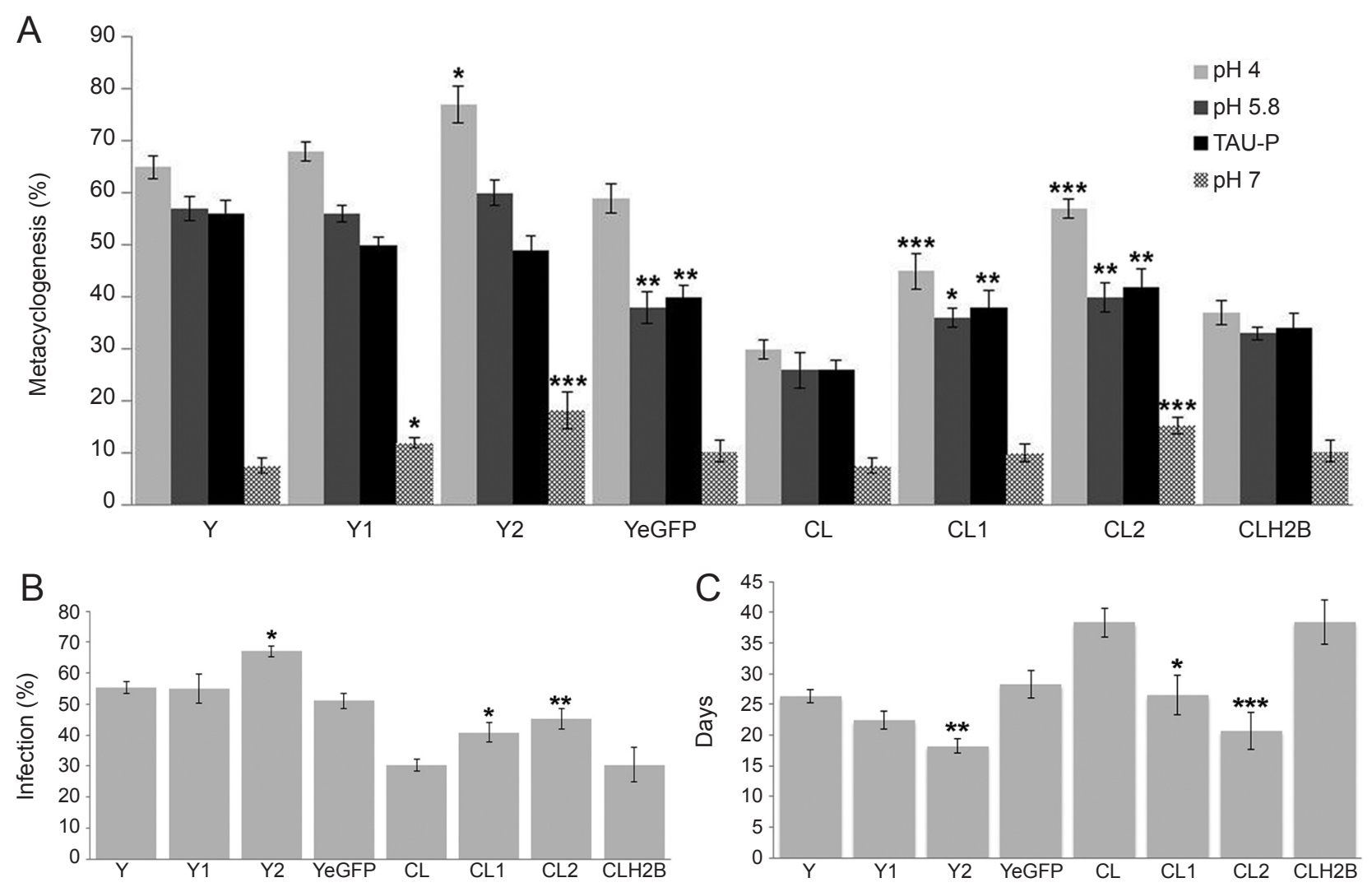

Fig. 1. Dynamics of in vitro cycling of staged of Trypanosoma cruzi. A - metacyclogenesis rates using neutral or low $\mathrm{pH}$ media or TAU-P. B - infection efficiency in Vero cells. C - time taken to complete a cycle. Names of individual strains - see the text. Significance levels were indicated with asterisks, and non-significant values were abbreviated NS.

in TAU-P. Interestingly, Y strain expressing the histone $\mathrm{H} 2 \mathrm{~B}$ showed a significant decrease in the metacyclogenesis rate at pH 5.8 and also in TAU-P. This phenomenon could be attributed to some effect associated to overexpression of the histone. In both media differentiation rates were slightly lower than those obtained at $\mathrm{pH} 4$ (Fig. 1A).

Using LIT or BHT medium at neutral $\mathrm{pH}$ spontaneous metacyclogenesis continuously increases between cycles from $7.7 \%(\mathrm{SD} \pm 0.9)$ to $18.3 \%(\mathrm{SD} \pm 3.2, \mathrm{Y} 2)$ and from $7.5 \%(\mathrm{SD} \pm 1.1, \mathrm{CL} 0)$ to $15.3 \%(\mathrm{SD} \pm 1.5, \mathrm{CL} 2)$.

Infection assays were performed 8 days after culture at $\mathrm{pH}$ 4. The first trypomastigotes were observed about 20 days post-infection in both strains, without subculturing. The optimal period of time for infective forms collection was 4-12 days after infection. Amastigotes were obtained by leaving the infected culture for 3-4 days (until 12-16 day post-infection) at $37^{\circ} \mathrm{C}$ without replacement of media.

When a large number of trypomastigotes (about $10^{7}$ cells) were released, they were collected and placed in $5 \mathrm{ml}$ of LIT or BHT medium at $\mathrm{pH}$ 7. Parasites were grown without subculturing and were observed periodically under the light microscope. Initially, they adopted a round shape and agglutinated forming of groups parasites groups; afterwards, they started to develop flagella and change to epimastigotes. The first epimastigotes were observed around 30 days post-inoculation. When parasites reached a density of approximately $10^{7}$ cells $/ \mathrm{ml}$, subcultures were performed. After several passages in LIT or BHT pH 7, they started growing similarly as cultured epimastigotes. The whole procedure described up to this point is what was called the 'first cycle' (Y1 and CL1).

Cycled epimastigotes $\left(10^{7}\right.$ cells $)$ of both strains (Y1 and CL1) were transferred to low $\mathrm{pH}$ media ( $\mathrm{pH} 4)$, for posterior infection repeating the above protocol, starting the 'second cycle' (Y2 and CL2). Comparing with previous cycles, the number of metacyclic trypomastigotes showed a significant increase and, additionally, infection times presented a significant decrease (Fig. 1C). Re-infection and cycling times diminished in each additional cycle. This might be because of selective pressures; the most virulent parasites are probably selected in each round of infection. The high differentiation percentages obtained for the second cycle of the CL Brener strain (about 60\% after ten days of culture in LIT medium at $\mathrm{pH}$ 7) reinforce this hypothesis. There was no need for low $\mathrm{pH}$ treatment for metacyclogenesis and these parasites were capable of infecting cells in 14 days. Both strains improved the metacyclogenesis rate (Fig. 1A), infectivity (Fig. 1B) and cycling time (Fig. 1C) after each cycle. Additional cy- 
Table 1. Rate of trypomastigotes and amastigotes of Trypanosoma cruzi per cell.

Trypomastigotes/cell

\begin{tabular}{lccccccccc}
\hline & Mean \pm SD & Y1 & Y2 & YH2B & CL0 & CL1 & CL2 & CLeGFP \\
\hline Y0 & $0.21 \pm 0.07$ & NS & $* * *$ & NS & NS & - & - & - \\
Y1 & $0.27 \pm 0.08$ & & - & - & - & NS & - & - \\
Y2 & $0.45 \pm 0.11$ & & & - & - & & $* * *$ & - \\
YH2B & $0.17 \pm 0.04$ & & & & - & - & - & NS \\
CL0 & $0.17 \pm 0.05$ & & & & & $* * *$ & $* * *$ & NS \\
CL1 & $0.29 \pm 0.08$ & & & & & & - & - \\
CL2 & $0.32 \pm 0.08$ & & & & & & & - \\
CLeGFP & $0.18 \pm 0.06$ & & & & & & & \\
\hline
\end{tabular}

Amastigotes/cell

\begin{tabular}{lcccccccc}
\hline & Mean \pm SD & Y1 & Y2 & YH2B & CL0 & CL1 & CL2 & CLeGFP \\
\hline Y0 & $16.3 \pm 2.2$ & $* * *$ & $* * *$ & NS & $* *$ & - & - & - \\
Y1 & $20.7 \pm 2.8$ & & - & - & - & NS & - & - \\
Y2 & $26.0 \pm 1.8$ & & & - & - & & $* * *$ & - \\
YH2B & $13.7 \pm 2.5$ & & & & - & - & - & NS \\
CL0 & $19.7 \pm 3.0$ & & & & & $* * *$ & $* * *$ & NS \\
CL1 & $21.8 \pm 2.1$ & & & & & & - & - \\
CL2 & $15.3 \pm 2.2$ & & & & & & & - \\
CLeGFP & $15.2 \pm 1.9$ & & & & & & & \\
\hline
\end{tabular}

NS not significant; SD standard deviation; ** $\mathrm{p}<0.05 ; * * * \mathrm{p}<0.005$.

cles further increase the mentioned parameters (data not shown). Subcultured epimastigotes that were previously submitted to this cycling protocol retained their enhanced infectivity for at least six months.

To obtain different $T$. cruzi stages from transgenic parasites, cycled epimastigotes of the CL Brener and Y strains were transfected with standard T. cruzi expression plasmids. To evaluate their capacity of expressing and targeting proteins to subcellular organelles in infective stages, we used the pTEX-H2B::eGFP and pTEX-eGFP vectors expressing the histone $\mathrm{H} 2 \mathrm{~B}$ fused to GFP (nuclear marker) and GFP alone (cytosolic marker). After selection parasites were challenged to low $\mathrm{pH}$ conditions. The number of metacyclic trypomastigotes was similar to the ones observed for non-transfected parasites. In infection assays, the first trypomastigotes were observed 30 days post-infection. Both trypomastigotes and amastigotes from the CL Brener and Y strains expressed fluorescent proteins (Fig. 2A,B).

Posterior re-infections, in which similar transgene expression levels were maintained, were achieved. When the amount of trypomastigotes was considerably high, they were shifted to LIT medium $\mathrm{pH} 7$ and, 30 days post-inoculation, the first epimastigotes, which still expressed the transgenes, were observed. Furthermore, Fig. 2C shows CL Brener parasites transfected with pTEX-H2B::eGFP, presenting an unusual morphology obtained 4-6 days post-infection between the trypomastigote/amastigote transition. This intermediate stage is similar to the previously described 'intracellular epimastigote-like form' (Almeida-de-Faria et al. 1999).

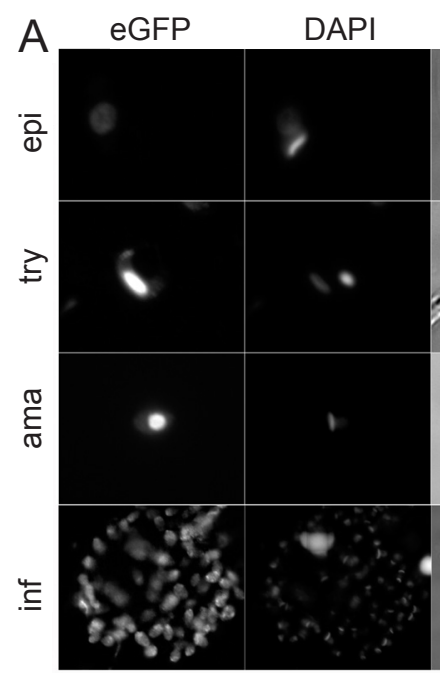

DIC Merge

B eGFP

DAPI

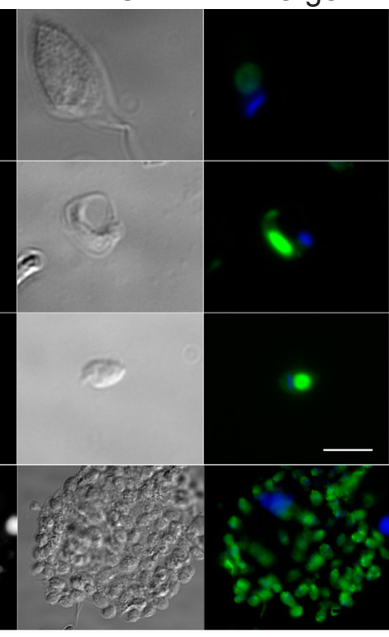

$\overline{\frac{0}{0}}$

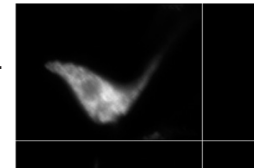

$\geqq$

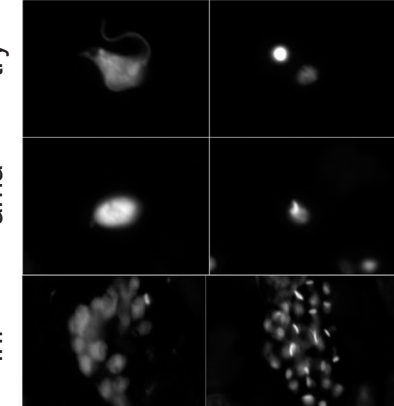

DIC

Merge

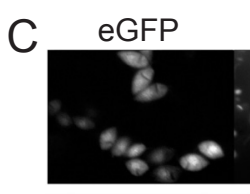

DAPI
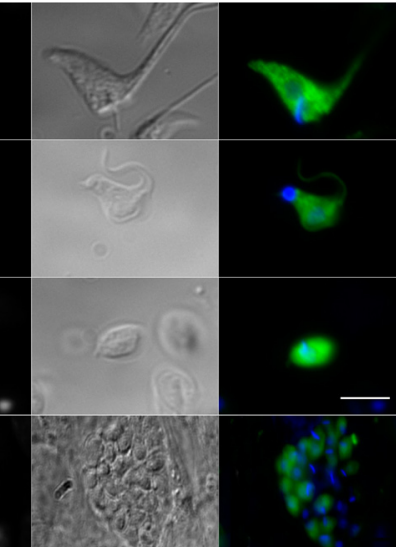

DIC

Merge

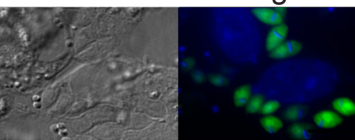

D

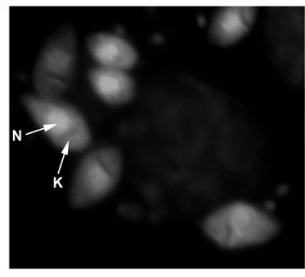

Fig. 2. Differentiation and infection using eGFP::H2B and eGFP transgenic parasites. CL Brener parasites transfected with the pTEX-eGFP::H2B (A) or Y strain parasites expressing the pTEX-eGFP (B) were analysed by fluorescence microscopy. Green fluorescence protein (eGFP), 4',6-diamidino-2-phenylindole (DAPI), differential interference contrast (DIC), and the merged image (Merge) shown in epimastigotes (epi), trypomastigotes (try), amastigotes (ama) and infected Vero cells (inf). C - Intracellular epimastigotes-like forms obtained at 4-6 days post-infection of Vero cells with CL Brener parasites carrying the pTEX-eGFP::H2B construction. D - magnification of intracellular epimastigotes; the typical nuclear $(\mathrm{N})$ and kinetoplast (K) organization of epimastigote forms is highlighted (arrows). Scale bars in the corner of the merged images $=5 \mu \mathrm{m}$. 
Finally, the number of trypomastigotes and amastigotes per cell were calculated. As Table 1 shows, a significant increase was observed in the amount of trypomastigotes and amastigotes per cell, when compared to controls (cycle 0 ), in both parasite strains during the cycle 2, and only in the Y strain during the cycle 1. On the other hand, the $Y$ strain has shown a significant increase in the amount of trypomastigotes and amastigotes per cell after the second cycle when compared with the CL Brener strain.

In conclusion, we were able to mimic $T$. cruzi life cycle in vitro using a simple method. Parasite recycling enhanced infectivity due to possible natural selection pressures. Furthermore, this method allows infection with transgenic parasites, which is a very powerful tool for studying protein localization and function in the infective parasite stages. In addition, fluorescent parasites can be used to monitor and record infection in vivo under a fluorescence microscope, allowing further advances in the understanding of the invasion process. Finally, in transgenic parasites where gene over-expression is partially toxic, antibiotic selection pressure must be maintained. For this reason, our design allows the use of antibiotics at every stage of the life cycle as a consequence of achieving infections in a G418 resistant Vero cell line. Nowadays, most of the functional genomics research on T. cruzi is made in epimastigote cells, mainly for technical simplicity of their culture and manipulation. We expect that the presented methodology allows and encourages experimentation with trypomastigotes and amastigotes, the most relevant life cycle stages from a therapeutic point of view.

Acknowledgments. This work was supported by Consejo Nacional de Investigaciones Científicas y Técnicas (CONICET, PIP 2010-0685 and 2011-0263), Agencia Nacional de Promoción Científica y Tecnológica (FONCYT PICT 2008-1209 and 2010-0289), and Fundación Bunge y Born (grant 2012). CAP and MRM are members of the career of scientific investigator and MdMC and LAB are research fellows from CONICET.

\section{REFERENCES}

Almeida-De-Faria M., Freymuller E., Colli W., Alves M.J. 1999: Trypanosoma cruzi: characterization of an intracellular epimastigote-like form. Exp. Parasitol. 92: 263-274.

CAmargo E.P. 1964: Growth and Differentiation in Trypanosoma cruzi. I. Origin of metacyclic trypanosomes in liquid media. Rev. Inst. Med. Trop. Sao Paulo. 6: 93-100.

Castellani O., Ribeiro L.V., Fernandes J.F. 1967: Differentiation of Trypanosoma cruzi in culture. J. Protozool. 14: 447-451.

Contreras V.T., Navarro M.C., De Lima A.R., Arteaga R., Duran F., Askue J., Franco Y. 2002: Production of amastigotes from metacyclic trypomastigotes of Trypanosoma cruzi. Mem. Inst. Oswaldo Cruz. 97: 1213-1220.

Contreras V.T., Salles J.M., Thomas N., Morel C.M., GoldENBERG S. 1985: In vitro differentiation of Trypanosoma cruzi under chemically defined conditions. Mol. Biochem. Parasitol. 16: 315-327.

De Lima A.R., Navarro M.C., Arteaga R.Y., Contreras V.T. 2008: Cultivation of Trypanosoma cruzi epimastigotes in low glucose axenic media shifts its competence to differentiate at metacyclic trypomastigotes. Exp. Parasitol. 119: 336-342.

DusAnic D.G. 1980: In vitro production of metacyclic trypomastigotes of Trypanosoma cruzi. J. Parasitol. 66: 1046-1049.

Goldenberg S., Avila A.R. 2011: Aspects of Trypanosoma cruzi stage differentiation. Adv. Parasitol. 75: 285-305.

Kimura E., Lay W.H., Fernandes J.F. 1978: Extracellular 'in vitro' evolution of metacyclic trypomastigotes isolated from
Trypanosoma cruzi cultures. Rev. Inst. Med. Trop. Sao Paulo. 20: $133-138$.

Kolev N.G., Ramey-Butler K., Cross G.A., Ullu E., Tschudi C. 2012: Developmental progression to infectivity in Trypanosoma brucei triggered by an RNA-binding protein. Science 338: 1352-1353.

Navarro M.C., De Lima A.R., Askue J., Contreras V.T. 2003: Morphological comparison of axenic amastigogenesis of trypomastigotes and metacyclic forms of Trypanosoma cruzi. Mem. Inst. Oswaldo Cruz. 98: 83-91.

Pereira C.A., Alonso G.D., Ivaldi S., Silber A.M., Alves M.J., Torres H.N., Flawia M.M. 2003: Arginine kinase overexpression improves Trypanosoma cruzi survival capability. FEBS Lett. 554: 201-205.

Rondinelli E., Silva R., Carvalho J.F., De Almeida Soares C.M., De Carvalho E.F., De Castro F.T. 1988: Trypanosoma cruzi: an in vitro cycle of cell differentiation in axenic culture. Exp. Parasitol. 66: 197-204.

Steverding D. 2008: The history of African trypanosomiasis. Parasit. Vectors 1: 3.

Tomlinson S., Vandekerckhove F., Frevert U., Nussenzweig V. 1995: The induction of Trypanosoma cruzi trypomastigote to amastigote transformation by low pH. Parasitology 110: 547-554.

Ucros H., Granger B., Krassner S.M. 1983: Trypanosoma cruzi: effect of $\mathrm{pH}$ on in vitro formation of metacyclic trypomastigotes. Acta Trop. 40: 105-112.

Accepted 6 May 2013 